\title{
Cerebral microbleeds and hemodynamic changes in individuals with cognitive impairment
}

\author{
${ }^{1}$ Eun-Ye Lim $M D,{ }^{2}$ Dong-Won Yang $M D P h D,{ }^{1}$ A-Hyun Cho $M D P h D,{ }^{3}$ Yong S. Shim $M D P h D$ \\ ${ }^{1}$ Department of Neurology, Yeouido St. Mary's Hospital, College of Medicine, The Catholic University \\ of Korea, Seoul; ${ }^{2}$ Department of Neurology, Seoul St. Mary's Hospital, College of Medicine, The \\ Catholic University of Korea, Seoul; ${ }^{3}$ Department of Neurology, Eunpyeong St. Mary's Hospital, \\ College of Medicine, The Catholic University of Korea, Seoul, Republic of Korea
}

\begin{abstract}
Background \& Objective: Cerebral microbleeds (CMBs) are considered key markers of small vessel pathology linking mechanisms relating to ischemic and amyloid related vascular damage in Alzheimer disease (AD). We aim to investigate the differences of hemodynamic markers between patients with and without CMBs who presented with cognitive decline using transcranial Doppler (TCD) ultrasonography. Methods: We consecutively enrolled patients who were diagnosed with mild cognitive impairment (MCI) and probable AD dementia from May 2011 to December 2012. Using TCD ultrasonography, cerebrovascular reactivity (CVR) was evaluated with hyperventilation (HV) and breath-holding $(\mathrm{BH})$ tests in addition to mean flow velocity (MFV) and pulsatility index (PI) of the middle cerebral artery. The number and location of CMBs were visually analyzed by two independent neurologists. Results: A total of 134 patients (probable $\mathrm{AD}, \mathrm{n}=81$; MCI, $\mathrm{n}=53$ ) were enrolled in this study. Among them, 28 (20.9\%) patients had CMBs; 18 (64.3\%) lobar CMBs, 7 (25.0\%) non-lobar CMBs. Patients with CMBs showed reduced MFV, higher resistance index value, and lower CVR compared with patients without CMBs after adjusting for age, vascular risk factors, and white matter hyperintensity. Multiple regression models showed that PI was dependent on age and presence of CMBs in frontal and parietal areas. CVR was closely associated with WMH severity and presence of CMBs in temporal.

Conclusions: Our study showed that patients with CMBs had higher microvascular resistance and decreased cerebrovascular autoregulation compared to those without.
\end{abstract}

Keywords: Cerebral microbleeds, transcranial Doppler ultrasonography, cognitive dysfunction, Alzheimer disease

\section{INTRODUCTION}

Increasing evidence suggests that amyloids are necessary but insufficient elements in the broader cascade responsible for the clinical features of Alzheimer's disease (AD)., ${ }^{1,2}$ Additional factors might contribute to clinical onset and progression of $\mathrm{AD}$ independently and/or simultaneously with $\mathrm{A} \beta$ and tau pathologies. ${ }^{3}$ Neuropathological studies showed that up to $84 \%$ of subjects clinically diagnosed with $\mathrm{AD}$ dementia have cerebrovascular pathology in addition to $\mathrm{AD}$ pathology. 4,5 Systematic reviews and metaanalyses have also highlighted the important role of cerebrovascular disease, especially cerebral small vessel disease, in $\mathrm{AD}$ onset and progression. ${ }^{4,6-8}$

Cerebral microbleeds (CMBs) are defined as small, round, hypointense lesions detected using gradient-echo (GRE) T2*-weighted or susceptibility-weighted imaging (SWI) of magnetic resonance imaging (MRI) sequences. ${ }^{9}$ CMBs are classified into two types of pathological changes based on location. Deep and infratentorial CMBs supposedly result from underlying hypertensive vasculopathy, while lobar CMBs are thought to reflect underlying cerebral amyloid angiopathy (CAA) ${ }^{10,11}$ This makes CMBs especially relevant in the context of $\mathrm{AD}$, in which diseases both amyloid pathology (including amyloid deposition in parenchyma and in vessels) and ischemic vascular pathology play a role. ${ }^{12} \mathrm{CMBs}$ are known to be associated with worse cognitive function and the patients with CMBs have higher incidence of dementia. ${ }^{13,14}$ Mechanisms by which CMBs influence cognitive

Address correspondence to: Yong S. Shim, MD, PhD, Department of Neurology, Eunpyeong St. Mary's Hospital, College of Medicine, The Catholic University of Korea, 1021, Tongil-ro, Eunpyeong-gu, Seoul, 03312, Republic of Korea. Tel.: 82-2-2030-4418, E-mail: ysshim @ catholic.ac.kr

Date of Submission: 18 February 2021; Date of Acceptance: 23 June 2021

https://doi.org/10.54029/2021hpr 
function remain controversial. We assumed that the presence of CMBs could exacerbate neurovascular dysfunction and further adversely affect clinical deterioration via hemodynamic changes. However, there are few studies on how $\mathrm{CMB}$ affects hemodynamic changes in patients with cognitive impairment.

Transcranial Doppler (TCD) ultrasonography is a non-invasive imaging technique widely used for investigation of cerebrovascular hemodynamics in the major cerebral arteries. Previously, we confirmed the usefulness of TCD ultrasonography to view early hemodynamic changes in subjects with cognitive impairment ${ }^{15}$ and showed that these hemodynamic changes were closely associated with cognitive decline. ${ }^{16}$

In this context, we aim to investigate the differences of hemodynamic markers between patients with and without CMBs who have cognitive impairment using TCD ultrasonography. Furthermore, we investigated whether the topographical location of CMBs could have different effects on hemodynamic factors.

\section{METHODS}

We consecutively enrolled subjects who visited the Department of Neurology at Bucheon St.Mary's hospital with memory complaints and were diagnosed with mild cognitive impairment(MCI) or probable AD dementia between May 2011 and December 2012. All participants received a medical history interview, physical and neurological examinations, screening laboratory tests including of vitamin B12, folate levels, and syphilis serology, and thyroid function tests. Baseline neuropsychological assessment was assessed using the Korean version of the Mini-Mental State Examination (K-MMSE) ${ }^{17}$ and functional performance was assessed with Clinical Dementia Rating (CDR) and CDR Sum of Boxes (CDR-SOB) scores. We used Petersen's criteria to diagnose MCI: patients with objective cognitive impairment less than 1.5 SD of the norm in at least 1 memory test, but normal activities of daily living (ADL) performance..$^{18}$ Probable $\mathrm{AD}$ dementia patients were those who fulfilled criteria proposed by the National Institute of Neurological and Communicative Disorders and Stroke and the AD and Related Disorders Association (NINCDS-ADRDA). ${ }^{19}$

Subjects also underwent 3-T brain MRI (Intera; Philips Medical Systems, Best, The Netherlands), including SWI, as well as conventional T1weighted, T2-weighted, and fluid-attenuated inversion recovery (FLAIR) sequences. The following parameters were used for the T2* SWI images: axial slice thickness, $5.0 \mathrm{~mm}$; inter-slice thickness, $2.7 \mathrm{~mm}$; repetition time (TR), $25 \mathrm{~ms}$; echo time (TE) $7.5 \mathrm{~ms}$; flip angle, $6^{\circ}$; matrix size, $384 \times 150$ pixels. Magnetic resonance angiography (MRA) was performed to exclude the presence of intracranial artery stenosis that might interfere with hemodynamic status. Participants were excluded if they showed large territorial infarcts or multiple lacunae on MRI or with carotid artery stenosis $>50 \%$ reduction in lumen diameter were excluded.

\section{Image analysis}

All MRI images were analyzed by two neurologists blinded to clinical information. CMBs were defined as small hypointense lesions within the brain parenchyma that measured $<10 \mathrm{~mm}$ on SWI images. CMB mimics such as calcification, cerebral venules, blood vessels, and cavernous malformation were not considered CMB. The locations of microbleeds were subdivided into the following categories: deep, if located in the basal ganglia, thalami, brain stem, or cerebellum; lobar, if located in one of the four cerebral lobes: frontal, parietal, occipital, and temporal; mixed, in the case of CMBs distributed in both lobar and non-lobar locations..$^{20}$ The total number of CMBs in each location was recorded. WMHs were visually rated on axial FLAIR images using the Fazekas scale. ${ }^{21}$ Lacunar infarcts were defined as lesions $<15 \mathrm{~mm}$ in diameter, with a low signal on T1-, a high signal on T2-weighted images, and a perilesional halo on FLAIR. ${ }^{22}$ The number of lacunar infarcts was also counted. Inter-rater agreement was excellent (weighted $\mathrm{k}$ and intraclass correlation coefficient $>0.90$ ) for all markers.

\section{TCD measurement}

All participants underwent evaluation using TCD ultrasonography. TCD examination was performed using a 2-MHz Doppler probe (Viasys Healthcare, Model Sonara) through the temporal bone window by a sonographer who was blinded to clinical diagnosis. The participants were placed in the supine position, and the TCD probe was fixed on the temporal window. The proximal segments of the MCA at 55-65 mm depths were examined bilaterally, and the mean flow velocity (MFV) was evaluated. The changes in MCA have been shown to reliably correlate with changes in cerebral blood flow. ${ }^{16}$ In addition, pulsatility index (PI) and resistance index (RI) were calculated. PI 
was calculated by subtracting the end-diastolic velocity from the peak systolic velocity and then dividing by the MFV. Thus, the PI is analogous to pulse pressure and recognized as a measure of distal flow resistance and vascular wall rigidity. The RI is a measure of peripheral flow resistance and was calculated by subtracting the end-diastolic velocity from the peak systolic velocity and then dividing by the peak systolic velocity.

After baseline assessments, cerebrovascular reactivity (CVR) was evaluated using hyperventilation (HV) and breath-holding $(\mathrm{BH})$ tests. In the first step, mean cerebral blood flow velocity was measured through bilateral temporal windows during normal breathing with the patient lying in the supine position. Next, participants were requested to begin HV with room air for approximately 2 minutes to reach a maximal decrease in flow velocity, and the MFV during the $\mathrm{HV}$ task (HV condition) was recorded. After confirming normal cerebral blood flow velocity, participants were requested to hold their breath for at least 30 seconds to achieve a maximal increase in flow velocity, and the MFV during the $\mathrm{BH}$ task (hypercapnic condition) was recorded. CVR was calculated as a percentage of baseline MFV and absolute changes by subtracting the baseline values from the maximum MFV during the $\mathrm{BH}$ task or the minimum MFV during the $\mathrm{HV}$ task as follows:

$\mathrm{CVR}_{\mathrm{BH}}=\left[\left(\mathrm{MFV}_{\mathrm{BH}^{-}}-\mathrm{MFV}_{\text {rest }}\right) / \mathrm{MFV}_{\text {rest }}\right] \times 100$
$\mathrm{CVR}_{\mathrm{HV}}=\left[\left(\mathrm{MFV}_{\text {rest }}-\mathrm{MFV}_{\mathrm{HV}}\right) / \mathrm{MFV}_{\text {rest }}\right] \times 100$

where $\mathrm{MFV}_{\mathrm{BH}}$ is the maximum MFV during the $\mathrm{BH}$ task, $\mathrm{MFV}_{\mathrm{HV}}$ is the minimum during the $\mathrm{HV}$ task, and $\mathrm{MFV}_{\text {rest }}$ is the resting (baseline) MFV in the middle cerebral artery.

\section{Statistical analysis}

To compare the baseline demographic characteristics between subjects with and without CMBs, $t$-tests tests were used for normally distributed data. For non-normal distribution of data, the Mann-Whitney test was used for continuous variables and Fisher's exact test for categorical variables. Analysis of covariance (ANCOVA) was used to compare baseline TCD parameters including MFV, PI, RI, and $\mathrm{CVR}_{\mathrm{BH} \text { or HV }}$ values between the subjects with and without CMBs, adjusted for age and WMH. In addition, we divided subjects with CMBs into two groups according to their location: lobar CMBs (with or without deep CMBs) and non-lobar CMBs, and compared TCD parameters between these two groups. The distribution of the dependent variables with a baseline RI and $\mathrm{CVR}_{\mathrm{BH}}$ was skewed, thus, a log-transformation (natural $\log$ ) was used to normalize the distribution.

The associations of hemodynamic factors and topographical CMBs were further evaluated using stepwise multiple linear regression analysis. Hemodynamic factors (MFV, PI, RI, and $\mathrm{CVR}_{\mathrm{BH} \text { and } \mathrm{HV}}$ ) were the dependent variables, and topographical CMBs were the independent variables of interest; adjustments for age and WMH were included in all models regardless of $\mathrm{P}$-value, and remaining variables were removed at $\mathrm{P}=0.10$.

All statistical analyses were performed using SPSS version 18 (SPSS, Chicago, IL, USA), and $\mathrm{p}<0.05$ was considered statistically significant.

\section{RESULTS}

A total of 134 patients were enrolled in this study: 53 patients (39.6\%) with MCI and $81(60.4 \%)$ with probable AD dementia. The mean age was 73.04 \pm 6.99 years, and female sex was predominant (72.2\%). Among participants, $28(20.9 \%)$ had CMBs; 11 (39.2\%) strictly lobar CMBs, 7 (25.0\%) strictly deep CMBs and 10 (35.7\%) mixed CMBs. The topographic distribution of CMBs was 7 patients $(33.3 \%)$ with at least $1 \mathrm{CMB}$ in the frontal lobe, $12(57.1 \%)$ in the temporal lobe, 10 $(47.6 \%)$ in the parietal lobe, $12(57.14 \%)$ in the occipital lobe, and $7(33.3 \%)$ in infratentorial and deep hemispheric regions.

Demographic characteristics based on the presence of CMBs are presented in Table 1. The patients with CMBs were older than the patients without $(75.46 \pm 5.4$ and $72.40 \pm 7.2, \mathrm{P}=0.038)$. Stroke history was more prevalent in patients with CMBs $(25.0 \%$ vs. $6.6 \%, \mathrm{P}=0.005)$. Statistically significant differences were not observed for sex, education years, and vascular risk factors. Baseline Mini-Mental State Examination (MMSE) score and dementia severity were also similar between the two groups. Regarding neuroimaging markers, the patients with CMBs showed higher burdens of WMH than patients without CMBs $(1.93 \pm$ 0.77 vs. $1.22 \pm 0.68, \mathrm{P}<0.001)$. The number of lacunes were also higher in the patients with than without $\mathrm{CMBs}(1.07 \pm 1.56$ vs. $0.27 \pm 0.62$, $\mathrm{P}<0.001)$.

The means of the baseline TCD parameters stratified based on CMB presence are shown in Table 2. The patients with CMBs showed reduced MFV $(44.95 \pm 17.2 v s .47 .70 \pm 12.3, \mathrm{P}=0.029)$, 
Table 1: Baseline demographics based on presence of CMBs

\begin{tabular}{|c|c|c|c|c|}
\hline Characteristics & Total $(n=134)$ & $\begin{array}{c}\text { CMB-negative } \\
\quad(n=106)\end{array}$ & $\begin{array}{c}\text { CMB-positive } \\
(\mathrm{n}=28)\end{array}$ & P-value ${ }^{1}$ \\
\hline Age, yrs & $73.04 \pm 6.99$ & $72.40 \pm 7.24$ & $75.46 \pm 5.41$ & $0.038^{*}$ \\
\hline No. (\%) female & $96(72.2)$ & $78(73.6)$ & $19(67.9)$ & 0.547 \\
\hline Education, yrs & $6.26 \pm 4.89$ & $6.28 \pm 4.89$ & $6.21 \pm 5.01$ & 0.975 \\
\hline MMSE & $19.08 \pm 5.91$ & $19.23 \pm 5.84$ & $18.54 \pm 6.26$ & 0.582 \\
\hline CDR & $0.88 \pm 0.72$ & $0.82 \pm 0.67$ & $1.09 \pm 0.89$ & 0.217 \\
\hline CDR-SOB & $4.74 \pm 4.27$ & $6.05 \pm 5.65$ & $6.05 \pm 3.77$ & 0.523 \\
\hline GDepS & $16.40 \pm 7.75$ & $16.54 \pm 7.88$ & $15.85 \pm 7.32$ & 0.683 \\
\hline APOE $\varepsilon 2$ carriers, No. (\%) & 7/116 (5.7) & 6/99 (6.1) & $1 / 25(4.0)$ & 0.690 \\
\hline APOE $\varepsilon 4$ carriers, No. (\%) & $39 / 116(31.7)$ & $35 / 99(35.4)$ & $4 / 25(16.0)$ & 0.063 \\
\hline \multicolumn{5}{|l|}{ Vascular risk factor } \\
\hline HTN, No. (\%) & $74(55.2)$ & $59(55.7)$ & $15(53.6)$ & 0.843 \\
\hline DM, No. $(\%)$ & $32(23.8)$ & $28(26.7)$ & $4(14.3)$ & 0.173 \\
\hline Dyslipidemia, No. (\%) & $18(13.43)$ & $14(13.2)$ & $4(14.3)$ & 0.882 \\
\hline IHD, No. (\%) & $11(8.2)$ & $6(5.7)$ & $3(10.7)$ & 0.342 \\
\hline Stroke Hx., No. (\%) & $14(10.44)$ & $7(6.6)$ & $7(25.0)$ & $0.005^{*}$ \\
\hline Smoking, No. (\%) & $10(7.4)$ & $6(5.7)$ & $4(14.3)$ & 0.122 \\
\hline WMH_Fazekas & $1.37 \pm 0.75$ & $1.22 \pm 0.68$ & $1.93 \pm 0.77$ & $<0.001 *$ \\
\hline Number of lacunas & $0.44 \pm 0.96$ & $0.27 \pm 0.62$ & $1.07 \pm 1.56$ & $<0.001 *$ \\
\hline
\end{tabular}

Abbreviations: MMSE, Mini-Mental State Examination; CDR, Clinical Dementia Rating; SOB, Sum of Boxes; GDepS, Geriatric Depression Scale; HTN, Hypertension; DM, Diabetes Mellitus; IHD, Ischemic Heart Disease; WMH, White Matter Hyperintensity

Values are presented as the mean \pm standard deviation (range) or number (percentage).

$P$-value ${ }^{l}$, CMBs negative vs CMBs positive

higher RI $(0.70 \pm 0.09 v s .0 .72 \pm 0.08, \mathrm{P}=0.012)$, and lower CVR $(0.31 \pm 0.98$ vs. $0.37 \pm 0.15, \mathrm{P}=$ 0.014 ) compared with the patients without CMBs after adjusting for age, vascular risk factors, and WMH. In addition, we analyzed again divided subjects with CMBs into two groups: lobar (with or without deep CMBs) and non-lobar CMBs. Subjects with lobar CMBs showed lower MFV $(42.58 \pm 13.5$ vs $52.06 \pm 25.2, \mathrm{p}=0.024)$, higher RI $(0.72 \pm 0.08$ vs $0.72 \pm 0.09, \mathrm{p}=0.009)$ and lower $\mathrm{CVR}_{\mathrm{HV}}(0.31 \pm 0.11$ vs $0.31 \pm 0.06, \mathrm{p}=0.018)$ than the subjects with non-lobar CMBs.

We also evaluated the associations of hemodynamic markers with topographical CMBs evaluated using multiple regression models (Table 3). PI was dependent on presence of CMBs in frontal $(\beta:-0.390, P=0.016)$ and parietal areas $(0.380, \mathrm{P}=0.019) . \mathrm{CVR}_{\mathrm{HV}}$ was closely associated with WMH severity $(-0.264$, $\mathrm{P}=0.004)$ and presence of CMBs in temporal $(0.396, \mathrm{P}=0.024)$ and, to some degree, occipital areas $(-0.326, \mathrm{P}=0.061)$.

\section{DISCUSSION}

In the present study, we found that the presence of CMBs was related to reduced cerebral blood flow, higher cerebral resistance, and decreased cerebrovascular reactivity in patients with cognitive impairment.

AD subjects with multiple CMBs have more severe cognitive impairment independently of disease duration ${ }^{23}$ and the presence of at least one $\mathrm{CMB}$ yielded a more than two folds increase risk of $\mathrm{AD}$ dementia in $\mathrm{MCI}$ patients. ${ }^{24}$ However, it remains unclear by which mechanisms of the pathological association between CMBs and cognitive function. Previous studies showed that reduced cerebral blood flow could affect the accumulation of amyloids and worsen the clinical features in the early stage of AD. ${ }^{25,26}$ Increased PI and impaired cerebral vasoreactivity also could contribute to the pathobiology of AD through resulting an insufficient blood supply to the brain. ${ }^{27,28}$ In this overall perspective, we assumed 
Table 2: Baseline TCD parameters stratified based on presence of CMBs

\begin{tabular}{|c|c|c|c|c|c|}
\hline & \multirow{2}{*}{$\begin{array}{c}\text { CMBs } \\
\text { Negative } \\
(n=106)\end{array}$} & \multicolumn{2}{|c|}{$\begin{array}{c}\text { CMBs Positive } \\
(n=28)\end{array}$} & \multirow{2}{*}{$P$-value ${ }^{I}$} & \multirow{2}{*}{$P$-value ${ }^{2}$} \\
\hline & & $\begin{array}{c}\text { Lobar CMBs }^{\text {a }} \\
(n=21)\end{array}$ & $\begin{array}{l}\text { Non Lobar } \\
\text { CMBs }(n=7)\end{array}$ & & \\
\hline \multirow{2}{*}{$\begin{array}{l}\text { Mean MFV } \\
(\mathrm{cm} / \mathrm{s})\end{array}$} & \multirow[t]{2}{*}{$47.70 \pm 12.28$} & \multicolumn{2}{|c|}{$44.95 \pm 17.17$} & $0.029 *$ & \\
\hline & & $42.58 \pm 13.54$ & $52.06 \pm 25.23$ & & $0.024 \dagger$ \\
\hline \multirow{2}{*}{ Mean PI } & \multirow[t]{2}{*}{$1.47 \pm 0.54$} & \multicolumn{2}{|c|}{$1.57 \pm 0.66$} & 0.098 & \\
\hline & & $1.62 \pm 0.70$ & $1.42 \pm 0.56$ & & 0.367 \\
\hline \multirow{2}{*}{ Mean RI } & \multirow[t]{2}{*}{$0.70 \pm 0.09$} & \multicolumn{2}{|c|}{$0.72 \pm 0.08$} & $0.012^{*}$ & \\
\hline & & $0.72 \pm 0.08$ & $0.72 \pm 0.09$ & & $0.009 \dagger$ \\
\hline \multirow{2}{*}{ Mean $\mathrm{CVR}_{\mathrm{BH}}$} & \multirow[t]{2}{*}{$0.49 \pm 0.14$} & \multicolumn{2}{|c|}{$0.50 \pm 0.14$} & 0.239 & \\
\hline & & $0.51 \pm 0.03$ & $0.55 \pm 0.15$ & & 0.487 \\
\hline \multirow{2}{*}{ Mean $\mathrm{CVR}_{\mathrm{HV}}$} & \multirow[t]{2}{*}{$0.37 \pm 0.15$} & \multicolumn{2}{|c|}{$0.31 \pm 0.98$} & $0.014^{*}$ & \\
\hline & & $0.31 \pm 0.11$ & $0.31 \pm 0.06$ & & $0.018 \dagger$ \\
\hline
\end{tabular}

Abbreviations: MFV, mean flow velocity; PI, pulsatility index; RI, resistance index; CVR, cerebrovascular reactivity; $\mathrm{BH}$, breathing holding index; HV, hyperventilation index

Values are presented as the mean \pm standard deviation.

TCD parameters(baseline $\mathrm{RI}, \mathrm{CVR}_{\mathrm{BH}}$ ) were subject to logarithmic transformation before proceeding to ANOVA with adjustments made for the covariates of age and $\mathrm{WMH}$.

${ }^{a}$ with mixed CMBs

$\boldsymbol{P}$-value $\boldsymbol{e}^{\boldsymbol{1}}$ CMBs negative vs CMBs positive

$\boldsymbol{P}$-value $\boldsymbol{e}^{2}$ Lobar/Mixed CMBs vs deep CMBs

Table 3: Associations between the location of CMBs and hemodynamic markers

\begin{tabular}{ccccc}
\hline Dependent & Independent variable & B & P-value & Partial R $^{2}$ \\
\hline PI & Age & & 0.040 & 0.040 \\
& CMBs_Frontal & -0.390 & 0.022 & \\
Mean CVR ${ }_{\mathrm{HV}}$ & CMBs_Parietal & 0.380 & 0.016 & \\
& & & 0.019 & 0.073 \\
& WMH & -0.264 & 0.004 & \\
\hline
\end{tabular}

Abbreviations: PI, pulsatility index; CVR, cerebrovascular reactivity; HV, hyperventilation index; CMBs, cerebral microbleeds; WMH, white matter hyperintensity 
that CMBs could affect deterioration of cerebral hemodynamics and aggravate accumulation of amyloid pathology and clinical deterioration in patients with $\mathrm{AD}$.

CMBs are representative neuroimaging markers of small vessel disease (SVD) in the brain. Accumulating evidence supported the important role of small vessel disease related vascular dysfunction in the initiation and progression of Alzheimer disease ${ }^{29,30}$ Possibly, amyloid $\beta$ (A $\beta$ ) deposition is mainly caused by a deficiency in the $A \beta$ clearance system rather than by an $A \beta$ overproduction $^{31}$, and $A \beta$ clearance is associated with the vascular system's integrity. ${ }^{30}$

SVD-related brain damage might be not confined to the visible lesion. The Rotterdam Scan Study revealed that people with CMBs, who have seemingly normal white matter of the brain, showed reduced white matter integrity within its microstructure.$^{32}$ In this scenario, a higher load of CMBs may indicate more extensive and severe subclinical microvascular damage, and their presence may influence cognition indirectly. ${ }^{33}$ Our results that showed hemodynamic changes in patients with CMBs supported these hypothesis.

We also found that these hemodynamic changes were prominent in the patients with lobar predominant $\mathrm{CMBs}$ than the patients with nonlobar CMBs. Lobar CMBs are thought to reflect underlying cerebral amyloid angiopathy (CAA) that is characterized by the deposition of $A \beta$ peptides in the small leptomeningeal and cortical vessels..$^{10} \mathrm{~A} \beta$ accumulation in vessel wall could lead to activation of vascular injury pathways (neurovascular dysfunction, BBB disruption, inflammation) that exacerbates neurodegenerative process and inflammatory response. ${ }^{34}$ Our results that the patients with lobar predominant CMBs showed hemodynamic deterioration than the patients with non-lobar CMBs are in line with previous pathological hypothesis that CMBs in $\mathrm{AD}$ could affect clinical prognosis via an independent pathway mediated by CAA-related vascular dysfunction, rather than a general vascular dysfunction pathway. ${ }^{16}$

In addition, we revealed that topographic distribution of CMBs had different effects on hemodynamic change; CMBs in frontal and parietal lobes mainly affected the change of PI, and CMBs in temporal and, to some degree, occipital lobes, were closely associated with cerebral vasoreactivity. Topographical distribution of CMBs and their effects on hemodynamic markers in subjects with cognitive impairment has only scarcely been studied.
CAA was known to favor posterior brain structures, especially the temporal and occipital lobe. ${ }^{35} \mathrm{~A} \beta$ deposition in the vessel wall could lead to obstruction of the vessel luminae and to the loss of vascular smooth muscle cells, resulting in impaired cerebral autoregulation. ${ }^{36}$ Our results supported the idea that CAA favor posterior brain structures, and especially the temporal and occipital lobe.

One study using transgenic mice to examine the effects of CAA showed different features of vascular dysfunction based on age. Cerebrovascular dysfunction could be rescued in the early phase of disease, but the beneficial effects were less evident in older mice when vascular damage has been established. ${ }^{37}$ Therefore, the presence of CMBs should be investigated when examining patients with cognitive impairment, and more active treatments to prevent and treat vascular disease and interventions to modify vascular dysfunction should be considered earlier in subjects with cognitive impairment. Furthermore, future therapeutic pharmacotherapies that would improve cerebral perfusion and increase cerebral vasoreactivity should be considered..$^{38}$

The present study had several limitations. First, the sample size for CMBs in various groups were relatively small, of which mixed CMBs accounted for a large proportion. So we included mixed CMBs as lobar CMBs in addition to strictly lobar CMBs. It is well known that CMBs have different underlying pathology depending on their location, and we wanted to investigate the hemodynamic markers according the location of CMBs. However, when we compared strictly lobar and deep CMBs excluding mixed CMBs, there was no significant value. The role of mixedlocation CMBs in cognitive impairment remain controversial. Recent study showed that mixedlocation CMBs was associated with a more severe burden of SVD, but not be driven by vascular risk factors alone. They suggested that the patients with mixed CMBs have both cerebral amyloid angiopathy and hypertensive angiopathy. ${ }^{39}$ Our results that showed pronounced hemodynamic changes in patients with lobar CMBs might be due to patients with mixed CMBs. A larger study including population with strictly divided into three groups of lobar, mixed and deep CMBs is needed to verify and extend the results of this population study. Second, the number of patients with CMBs and the differences of values of hemodynamic markers was so small, so it might be limitation to the clinical significance. However, it is meaningful that our study suggested one 
hypothesis that how CMBs affect pathologic changes in patients with cognitive impairment. Third, two methods $\left(\mathrm{CVR}_{\mathrm{HV}}, \mathrm{CVR}_{\mathrm{BH}}\right)$ were used to analyze cerebral vasoreactivity. $\mathrm{CVR}_{\mathrm{HV}}$ showed a significant value; however, $\mathrm{CVR}_{\mathrm{BH}}$, which is more widely used to evaluate CVR, did not. ${ }^{40} \mathrm{We}$ assumed that $\mathrm{BH}$ might be difficult for elderly patients, especially for patients with dementia, and HV-impaired vasoconstriction might be an earlier change than vasodilation. Fourth, the voluntary breathing and $\mathrm{HV}$ techniques have merit because they can be easily performed; however, the degree of irritation may not be constant. For more objective CVR measurements, additional imaging studies such as functional MRI (fMRI) and brain single-photon emission computed tomography would be helpful. However, in a previous study, a close correlation was observed between TCD measurement and fMRI ${ }^{41}$ Although imaging analysis may be more accurate than TCD measurement to detect cerebral vasoreactivity, TCD sonography could be more widely used in patients with dementia due to easier access.

Despite these limitations, our findings indicate that CMBs play important roles in hemodynamic changes in patients with cognitive decline. In particular, lobar CMBs in posterior brain structures could play more detrimental roles in cerebral hemodynamics. A prospective study with larger sample sizes would be required to strengthen our results.

\section{DISCLOSURE}

Ethics statement: The present study protocol was reviewed and approved by the institutional review board of Yeouido St. Mary's Hospital, The Catholic University of Korea. The board exempted informed consent due to retrospective study.

Financial support: None

Conflicts of interest: None

\section{REFERENCES}

1. Jack CR, Jr., Vemuri P. Amyloid-beta - a reflection of risk or a preclinical marker? Nat Rev Neurol 2018;14:319-20.

2. Dubois B, Epelbaum S, Nyasse F, et al. Cognitive and neuroimaging features and brain beta-amyloidosis in individuals at risk of Alzheimer's disease (INSIGHTpreAD): a longitudinal observational study. Lancet Neurol 2018;17:335-46.

3. Jack CR, Jr., Bennett DA, Blennow K, et al. NIAAA Research Framework: Toward a biological definition of Alzheimer's disease. Alzheimers Dement 2018;14:535-62.
4. Arvanitakis Z, Capuano AW, Leurgans SE, et al. Relation of cerebral vessel disease to Alzheimer's disease dementia and cognitive function in elderly people: a cross-sectional study. Lancet Neurol 2016;15:934-43.

5. Toledo JB, Arnold SE, Raible K, et al. Contribution of cerebrovascular disease in autopsy confirmed neurodegenerative disease cases in the National Alzheimer's Coordinating Centre. Brain 2013;136:2697-706.

6. Gorelick PB, Scuteri A, Black SE, et al. Vascular contributions to cognitive impairment and dementia: a statement for healthcare professionals from the american heart association/american stroke association. Stroke 2011;42:2672-713.

7. MarshallRS. Effects of altered cerebral hemodynamics on cognitive function. J Alzheimers Dis 2012;32:63342.

8. Sabayan B, Jansen S, Oleksik AM, et al. Cerebrovascular hemodynamics in Alzheimer's disease and vascular dementia: a meta-analysis of transcranial Doppler studies. Ageing Res Rev 2012;11:271-7.

9. Greenberg SM, Charidimou A. Diagnosis of cerebral amyloid angiopathy: Evolution of the Boston Criteria. Stroke 2018;49:491-7.

10. Greenberg SM, Vernooij MW, Cordonnier C, et al. Cerebral microbleeds: a guide to detection and interpretation. Lancet Neurol 2009;8:165-74.

11. Moran C, Phan TG, Srikanth VK. Cerebral small vessel disease: a review of clinical, radiological, and histopathological phenotypes. Int J Stroke 2012;7:3646.

12. Li H, Guo Q, Inoue $\mathrm{T}$, et al. Vascular and parenchymal amyloid pathology in an Alzheimer disease knock-in mouse model: interplay with cerebral blood flow. Mol Neurodegener 2014;9:28-.

13. Patel B, Lawrence AJ, Chung AW, et al. Cerebral microbleeds and cognition in patients with symptomatic small vessel disease. Stroke 2013;44:356-61.

14. Akoudad S, Wolters FJ, Viswanathan A, et al. Association of cerebral microbleeds with cognitive decline and dementia. JAMA Neurol 2016;73:934-43.

15. Alwatban M, Murman DL, Bashford G. Cerebrovascular reactivity impairment in preclinical Alzheimer's disease. J Neuroimaging 2019;29:493-8.

16. Charidimou A, Boulouis G, Gurol ME, et al.Emerging concepts in sporadic cerebral amyloid angiopathy. Brain 2017;140:1829-50.

17. Kang Y, Na DL, Hahn S. A validity study on the Korean Mini-Mental State Examination (K-MMSE) in dementia patients. J Korean Neurol Assoc 1997;15:300-8.

18. Petersen RC. Mild cognitive impairment as a diagnostic entity. J Intern Med 2004;256:183-94.

19. Dubois B, Feldman HH, Jacova C, et al. Research criteria for the diagnosis of Alzheimer's disease: revising the NINCDS-ADRDA criteria. Lancet Neurol 2007;6:734-46.

20. Gregoire SM, Chaudhary UJ, Brown MM, et al. The Microbleed Anatomical Rating Scale (MARS): reliability of a tool to map brain microbleeds. Neurology 2009;73:1759-66. 
21. Kapeller P, Schmidt R, Enzinger C, et al. CT and MRI rating of white matter changes. J Neural Transm Suppl 2002:41-5.

22. Wardlaw JM, Smith EE, Biessels GJ, et al. Neuroimaging standards for research into small vessel disease and its contribution to ageing and neurodegeneration. Lancet Neurol 2013;12:822-38.

23. Goos JD, Kester MI, Barkhof F, et al. Patients with Alzheimer disease with multiple microbleeds: relation with cerebrospinal fluid biomarkers and cognition. Stroke 2009;40:3455-60.

24. Staekenborg SS, Koedam EL, Henneman WJ, et al. Progression of mild cognitive impairment to dementia: contribution of cerebrovascular disease compared with medial temporal lobe atrophy. Stroke 2009;40:1269-74.

25. Wierenga CE, Hays CC, Zlatar ZZ. Cerebral blood flow measured by arterial spin labeling MRI as a preclinical marker of Alzheimer's disease. $J$ Alzheimers Dis 2014;42 Suppl 4:S411-9.

26. Roher AE, Debbins JP, Malek-Ahmadi M, et al. Cerebral blood flow in Alzheimer's disease. Vasc Health Risk Manag 2012;8:599-611.

27. Stefani A, Sancesario G, Pierantozzi M, et al. CSF biomarkers, impairment of cerebral hemodynamics and degree of cognitive decline in Alzheimer's and mixed dementia. J Neurol Sci 2009;283:109-15.

28. van Beek AH, Claassen JA, Rikkert MG, et al. Cerebral autoregulation: an overview of current concepts and methodology with special focus on the elderly. J Cereb Blood Flow Metab 2008;28:1071-85.

29. Iturria-Medina Y, Sotero RC, Toussaint PJ, et al. Early role of vascular dysregulation on late-onset Alzheimer's disease based on multifactorial datadriven analysis. Nat Commun 2016;7:11934.

30. Klohs J. An integrated view on vascular dysfunction in Alzheimer's disease. Neurodegene Dis 2019;19:10927.

31. Prasansuklab A, Tencomnao T. Amyloidosis in Alzheimer's Disease: The toxicity of amyloid beta $(A<i>\beta</ i>)$, Mechanisms of its accumulation and implications of medicinal plants for therapy. Evid Based Complement Alternat Med 2013;2013:413808.

32. Akoudad S, de Groot M, Koudstaal PJ, et al. Cerebral microbleeds are related to loss of white matter structural integrity. Neurology 2013;81:1930-7.

33. Litak J, Mazurek M, Kulesza B, et al. Cerebral small vessel disease. Int J Mol Sci 2020;21:9729.

34. Bell RD, Zlokovic BV. Neurovascular mechanisms and blood-brain barrier disorder in Alzheimer's disease. Acta Neuropathol 2009;118:103-13.

35. Attems J, Quass M, Jellinger KA, et al. Topographical distribution of cerebral amyloid angiopathy and its effect on cognitive decline are influenced by Alzheimer disease pathology. J Neurol Sci 2007;257:49-55.

36. van Dijk EJ, Prins ND, Vermeer SE, et al. Plasma amyloid beta, apolipoprotein E, lacunar infarcts, and white matter lesions. Ann Neurol 2004;55:570-5.

37. Park L, Koizumi K, El Jamal S, et al. Age-dependent neurovascular dysfunction and damage in a mouse model of cerebral amyloid angiopathy. Stroke 2014;45:1815-21.
38. Love S, Miners JS. Cerebrovascular disease in ageing and Alzheimer's disease. Acta Neuropathol 2016;131:645-58.

39. Blanc C, Viguier A, Calviere L, et al. Underlying small vessel disease associated with mixed cerebral microbleeds. Front Neurol 2019;10:1126.

40. Dumas A, Dierksen GA, Gurol ME, et al. Functional magnetic resonance imaging detection of vascular reactivity in cerebral amyloid angiopathy. Ann Neurol 2012;72:76-81.

41. Herrera CR, Beltramini GC, Avelar WM, et al. Cerebral vasomotor reactivity assessment using Transcranial Doppler and MRI with apnea test. Braz J Med Biol Res 2016;49:e5437. 